\title{
Synthesis of Boron Nanowires, Nanotubes, and Nanosheets
}

\author{
Rajen B. Patel, ${ }^{1}$ Tsengming $\mathrm{Chou}^{2}$ and Zafar Iqbal ${ }^{3}$ \\ ${ }^{1}$ Materials Science and Engineering, New Jersey Institute of Technology, Newark, NJ 07102, USA \\ ${ }^{2}$ Laboratory for Multiscale Imaging, Stevens Institute of Technology, Hoboken, NJ 07030, USA \\ ${ }^{3}$ Department of Chemistry and Environmental Science, New Jersey Institute of Technology, Newark, NJ 07102, USA \\ Correspondence should be addressed to Rajen B. Patel; rajenbp@gmail.com
}

Received 6 November 2014; Revised 17 December 2014; Accepted 17 December 2014

Academic Editor: Santanu K. Maiti

Copyright (C) 2015 Rajen B. Patel et al. This is an open access article distributed under the Creative Commons Attribution License, which permits unrestricted use, distribution, and reproduction in any medium, provided the original work is properly cited.

\begin{abstract}
The synthesis of boron nanowires, nanotubes, and nanosheets using a thermal vapor deposition process is reported. This work confirms previous research and provides a new method capable of synthesizing boron nanomaterials. The materials were made by using various combinations of $\mathrm{MgB}_{2}, \mathrm{Mg}\left(\mathrm{BH}_{4}\right)_{2}, \mathrm{MCM}-41, \mathrm{NiB}$, and Fe wire. Unlike previously reported methods, a nanoparticle catalyst and a silicate substrate are not required for synthesis. Two types of boron nanowires, boron nanotubes, and boron nanosheets were made. Their morphology and chemical composition were determined through the use of scanning electron microscopy, transmission electron microscopy, and electron energy loss spectroscopy. These boron-based materials have potential for electronic and hydrogen storage applications.
\end{abstract}

\section{Introduction}

Carbon nanotubes and graphene have been researched extensively for the past two decades due to their remarkable electrical, thermal, and mechanical properties [1-7], which surpass those of a wide range of materials. For this reason, carbon nanotube/graphene analogs involving other elements have been synthesized such as boron nitride [8-10], titanium dioxide [11], numerous sulfides [12-14], and zinc oxide [15]. A common trait that many of these materials possess is that they have a layered crystal structure. It would therefore be of interest to synthesize elements or compounds with layered nanostructures that do not form a layered structure in their bulk forms. These types of materials would require special consideration to determine how flat or rolled layers form during their synthesis.

Pure boron was reported to be stable in a nanotube configuration [16-18] and modeling of pure boron sheets (a graphene analog) has also been performed $[19,20]$. (Pure in this case simply means that the vast majority of the boron in the structure is not part of a compound.) Pure boron nanostructures could have properties unique in comparison to bulk boron phases, potentially making them useful for electronic and optical applications due to electron confinement effects similar to that in graphene and carbon nanotubes. Moreover, boron nanostructures would possess superior thermal and mechanical properties in comparison to their bulk analogs, much like in carbon and boron nitride nanostructures. From a purely theoretical perspective, understanding how a group 3 element such as boron can remain stable in a nanotube configuration is of great interest. The mechanism which keeps such a structure stable could be pertinent to the formation of other types of nanostructures using different elements or compounds. A novel process to grow boron nanowires and nanotubes is reported here. Additionally, the synthesis of a novel boron nanostructure, boron nanosheets, is described.

\section{Experimental}

A method to grow boron nanostructures was employed based on the procedure first used to synthesize boron nanotubes [16, 21]. However, there were some key differences. For example, $\mathrm{BCl}_{3}$ was not used because it is highly reactive; instead, solid boron precursors and catalyst were utilized in a solid-solid reaction process in flowing inert gas. First, $50 \mathrm{wt} \% \mathrm{MgB}_{2}$ (Alfa Aesar), $30 \mathrm{wt} \%$ nano- $\mathrm{NiB}$ (prepared in a manner following $[22,23])$, and $20 \mathrm{wt} \%$ of mesostructured hexagonal framework MCM-41 zeolite powder (Sigma-Aldrich) are mixed 


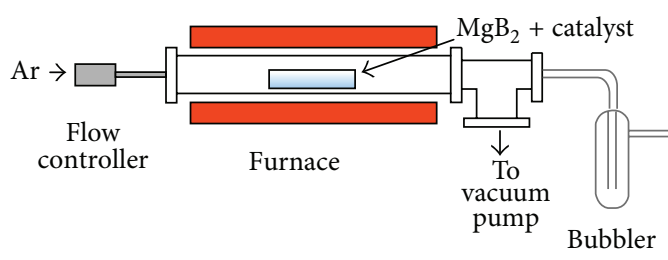

(a)

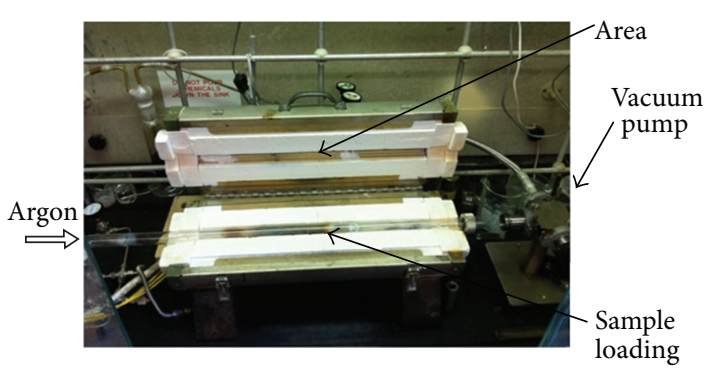

(b)

FIGURE 1: (a) Schematic diagram of the CVD system used in these experiments. (b) A photograph of the experimental apparatus [21].

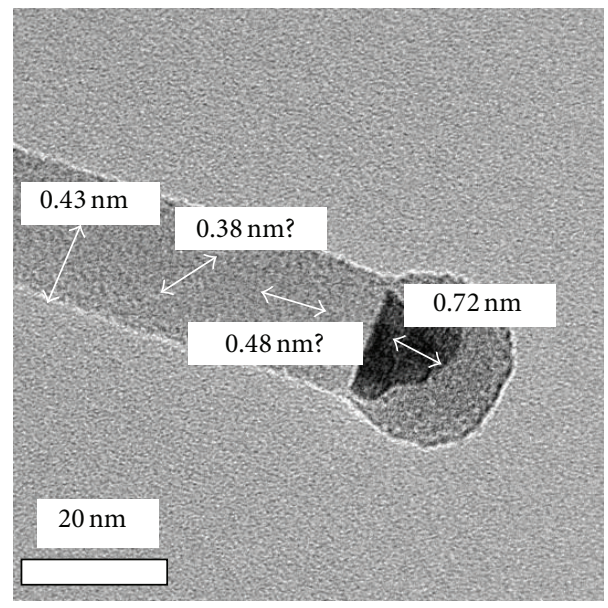

(a)

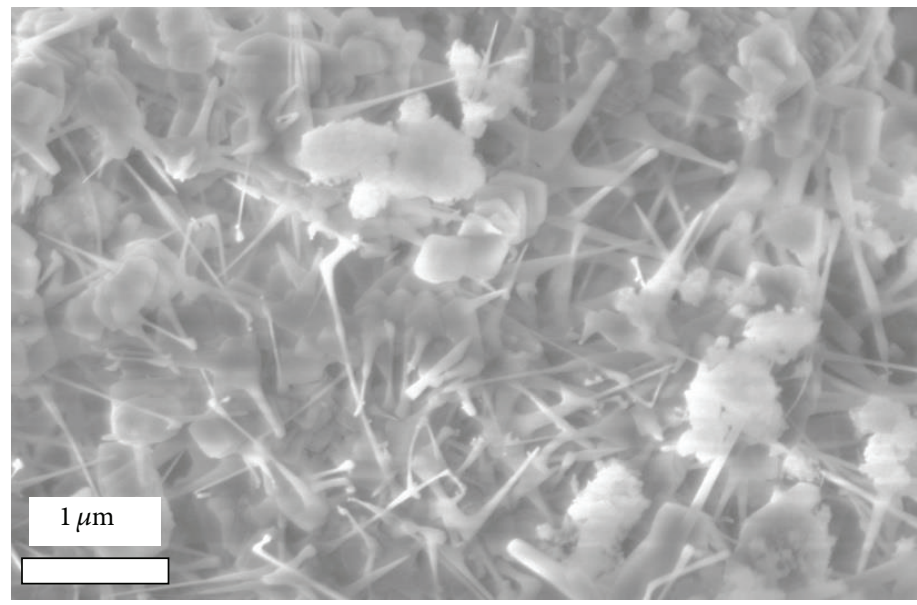

(b)

FIgURE 2: (a) TEM and (b) SEM images of boron nanowires synthesized using $\mathrm{MgB}_{2}$ and MCM-41.

and reduced in particle size by grinding using a mortar and pestle. Typically, 0.02-0.1 grams of the mixture was added and ground in an agate mortar for about an hour to ensure that the powder was well mixed. It was then ground further for several hours using a rotary mixer, which uses cylindrical ceramic pieces as milling media. Finally, the mixture was loaded into the quartz reactor of the chemical vapor deposition (CVD) apparatus shown in Figure 1.

The quartz tube was pumped down to $10^{-3}$ torr and heated to $950^{\circ} \mathrm{C}$ at a rate of $10^{\circ} \mathrm{C} / \mathrm{min}$ under flowing argon at $100 \mathrm{sccm}$ (standard cubic centimeters per minute). The temperature was held at $950^{\circ} \mathrm{C}$ for 60 minutes. After completion of the reaction, the furnace was switched off and the reaction tube was allowed to cool down to room temperature under flowing argon. Alternatively, one could use $\mathrm{Mg}\left(\mathrm{BH}_{4}\right)_{2}$ instead of $\mathrm{MgB}_{2}$, but then the optimal reaction temperature would be $800^{\circ} \mathrm{C}$. Another growth condition using $\mathrm{MgB}_{2}$ was also tested where an iron wire replaced nano- $\mathrm{NiB}$ as the catalyst and the MCM-41 template was not used. This was to test the importance of the catalyst and the template in the process reaction.

Scanning electron microscopy (SEM): SEM images were obtained with a VP-1530 Carl Zeiss LEO (Peabody, MA) field emission scanning electron microscope. The samples were mounted on aluminum stubs using double-sided carbon tape.
Scanning transmission electron microscopy (STEM): a FEI CM-20 FEG STEM equipped with a Gatan Enfina PEELS Spectrometer and an Oxford Max-80 SDD EDS system was used. Samples were suspended in ultrapure methanol at $1 \mathrm{wt} \%$ concentration, and a $1 \mu \mathrm{L}$ drop of the solution was placed on a lacey carbon TEM grid placed on a filter paper. The TEM grid was then placed in a vacuum oven to dry at $80^{\circ} \mathrm{C}$. Electron energy loss spectroscopy (EELS) analysis was performed in conjunction with STEM dark field imaging.

\section{Results and Discussion}

The boron nanowires (BNWs) synthesized are shown in Figures 2-4. BNWs are well known in the literature and have been shown to have useful characteristics [24-26] leading to applications for them in flexible electronics and field effect transistors. Figure 2 shows a TEM image of a boron nanowire prepared using $\mathrm{MgB}_{2}$ as the precursor. The nanowires have diameters in the $30-50 \mathrm{~nm}$ range, with lengths up to many microns. A catalyst particle is evident at the head of the nanowire indicating that the growth mechanism is through a vapor-liquid-solid (VLS) reaction process. In this sample, only nanowires were observed with no evidence of boron nanotubes or nanosheets. Fast Fourier transform (FFT) analysis 


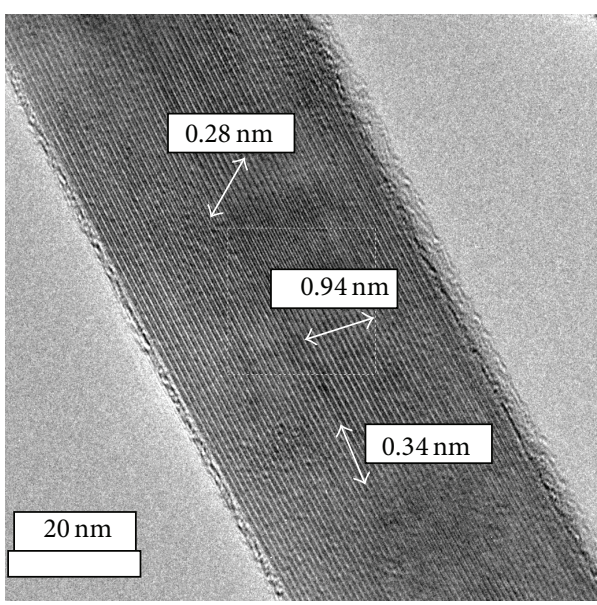

(a)

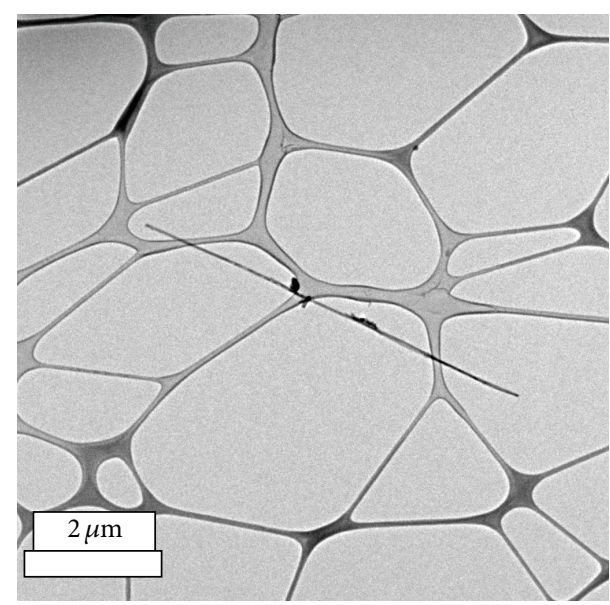

(b)

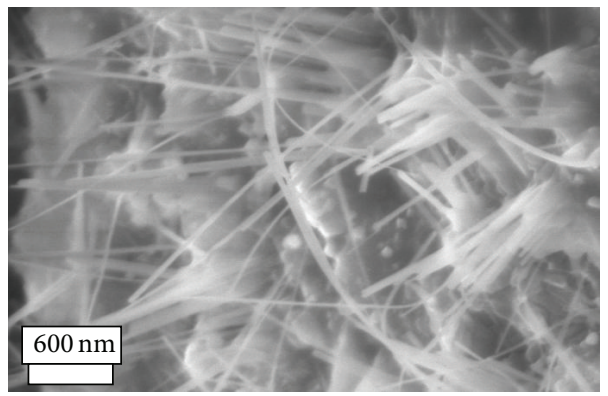

(c)

Figure 3: (a) High resolution TEM image of boron nanowire and (b) low magnification TEM image of the same nanowire showing length. (c) SEM image showing these nanowires can be obtained at high yield with consistent dimensions. Material was synthesized using $\mathrm{Mg}\left(\mathrm{BH}_{4}\right)_{2}$ and MCM-41.

of the nanowires indicated that they have a crystal spacing of $0.43 \mathrm{~nm}$. Spacing of $0.48 \mathrm{~nm}$ and $0.38 \mathrm{~nm}$ was also found, but due to lack of image resolution, the evidence for them is not conclusive. Note that a $0.38 \mathrm{~nm}$ spacing has been previously reported for boron nanowires [21]. EELS analysis confirmed that the nanowire consisted only of boron. The region around a nanowire head with the catalyst showed a crystal spacing of $0.72 \mathrm{~nm}$ using FFT analysis, indicating that the material surrounding the catalyst has a crystal structure that is different from that of the rest of the nanowire. An SEM image is included which shows that the nanowires can be made at high yield with consistent dimensions.

When the boron nanowires were grown using $\mathrm{Mg}\left(\mathrm{BH}_{4}\right)_{2}$ as the precursor, a different material is produced, as shown in Figure 3. Pure boron nanowires were found in the samples prepared, but they had extremely large aspect ratios, with lengths up to $50 \mu \mathrm{m}$ and diameters in the range of 30$50 \mathrm{~nm}$. Using FFT image analysis, crystal spacing of $0.94 \mathrm{~nm}$, $0.34 \mathrm{~nm}$, and $0.28 \mathrm{~nm}$ was found, indicating that these nanowires have a different crystal structure than those obtained using $\mathrm{MgB}_{2}$. Moreover, an extremely thin nanofibrous material was also found, as evident from Figure 4 . The nanofibrous material had lengths of roughly 100-200 $\mathrm{nm}$ and diameters close to $20 \mathrm{~nm}$. High resolution imaging and spectroscopic analysis could not, however, be carried out on this material because of its instability in the electron beam. These nanostructures therefore clearly exhibited a VLS type growth mechanism, and unlike other phases of boron, they showed significant curvature. They also appeared to be hollow, but this was difficult to confirm conclusively. These findings, therefore, indicate the formation of a boron nanotube as opposed to a nanowire, but improved data analysis would be needed to confirm this.

In another approach, the MCM-41 template was not used and the nano-NiB catalyst was replaced by an iron wire, but otherwise, the process was the same as that used with $\mathrm{MgB}_{2}$. In prior studies, it was assumed that MCM-41 was necessary as a template for the growth of boron nanotubes and that a nanoparticle catalyst was also crucial. SEM and STEM images from samples prepared using this method are shown in Figures 5-7. Here, both nanotubes and nanosheets were found among boron nanowires. The nanotubes were hollow in their interior and clearly had sidewalls. These two characteristics are critical, as they differentiate nanotubes from nanowires. The boron nanotubes showed a diameter of roughly $10 \mathrm{~nm}$ and lengths up to many microns. An individual nanotube depicted in Figure 6 clearly had two different diameters caused by variations in the growth conditions 


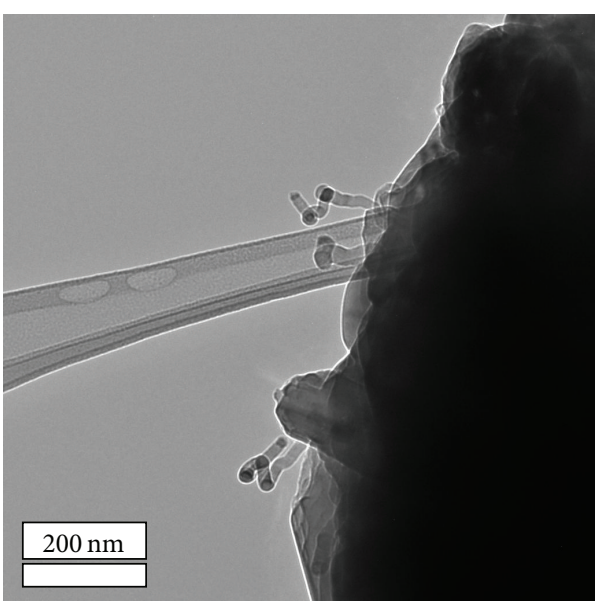

(a)

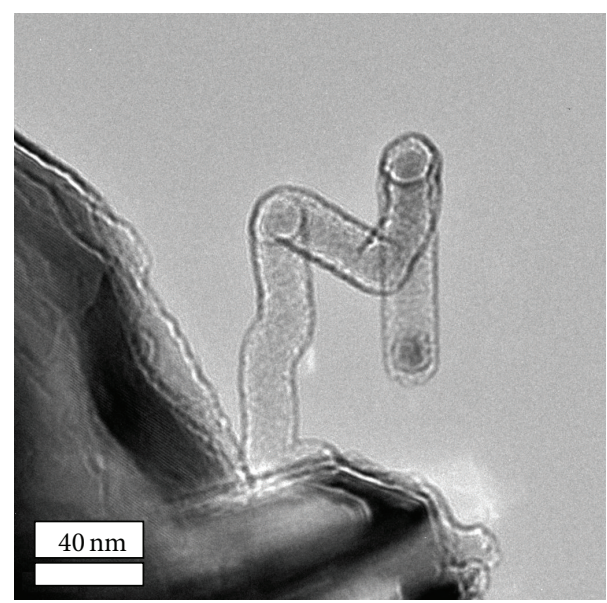

(b)

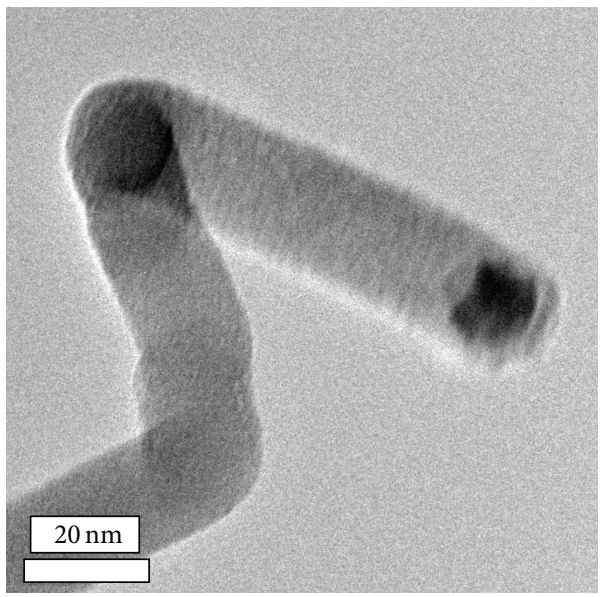

(c)

Figure 4: (a) Low magnification TEM image of thin nanofibers found in sample made with $\mathrm{Mg}\left(\mathrm{BH}_{4}\right)_{2}$ and MCM-41. (b) TEM image of a highly curved nanofiber showing a catalyst head indicating it grew using a VLS mechanism. Note the structure around the catalyst head is not bulbous or seemingly different from that of the rest of the nanofiber. (c) Another nanofiber exhibiting similar characteristics.

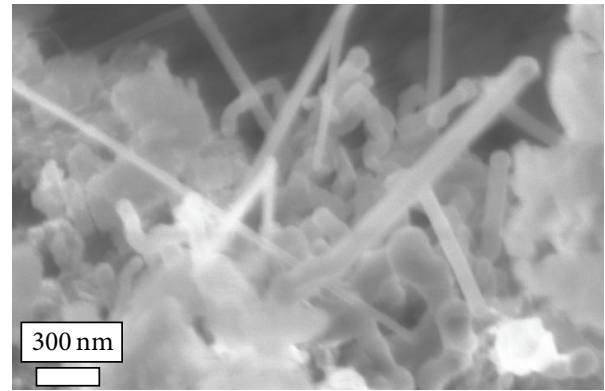

FIGURE 5: SEM image of product synthesized using $\mathrm{MgB}_{2}$, Fe Wire, and no MCM-41. Note that a consistent product at a high yield is obtained.

around the nanotube. Additionally, boron nanosheets, thin sheetlike nanostructures covering areas on the order of a number of square micrometers, were found in samples prepared by this method. The sheets were revealed to be amorphous by selected area electron diffraction.
To summarize, in comparison to well-known techniques, such as magnetron sputtering, to synthesize boron nanowires [24-26], the reported process has the added advantage of producing a wide variety of structures. Furthermore, because it is a thermal vapor deposition technique, there could be some advantages in scaling and flexibility. However, other methods are most likely more suited for producing well-aligned arrays of boron nanowires. The CVD method used here would have to be modified for producing aligned nanostructured arrays.

\section{Conclusions}

A novel method which would need future optimization is described which can grow boron nanotubes, boron nanosheets (a new material), and boron nanowires. This method is significant because $\mathrm{MCM}-41$, nanosized catalyst, and $\mathrm{BCl}_{3}$ gas are not required for the process. This greatly simplifies creating these boron nanomaterials and makes scale-up and future experimentation much more straightforward. These materials have not been characterized thoroughly, but they 


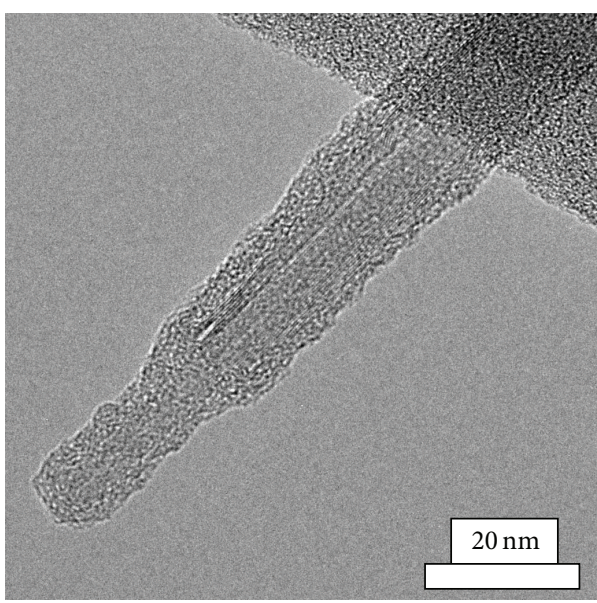

(a)

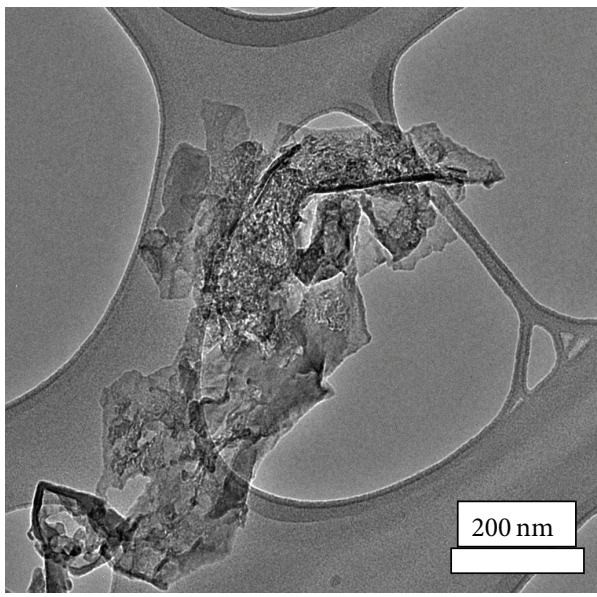

(c)

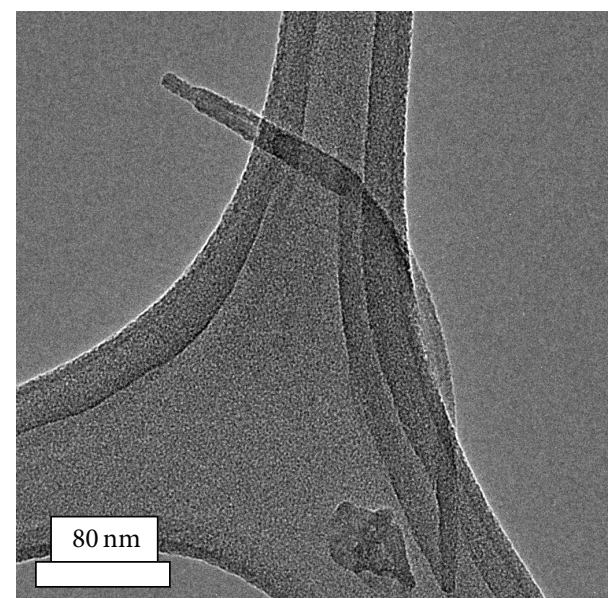

(b)

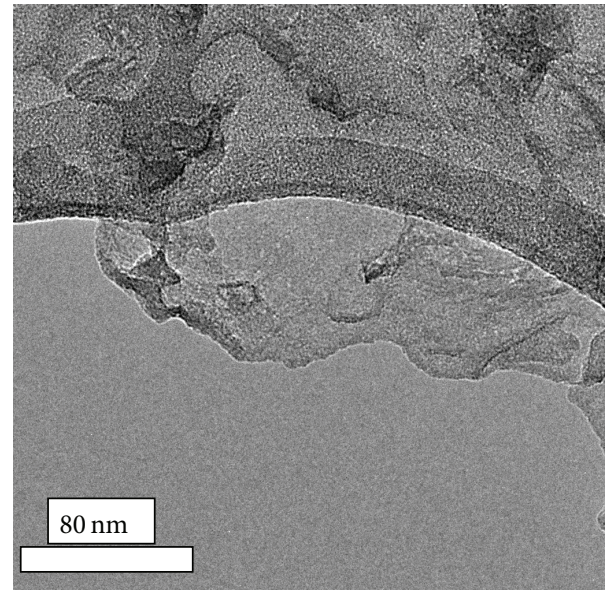

(d)

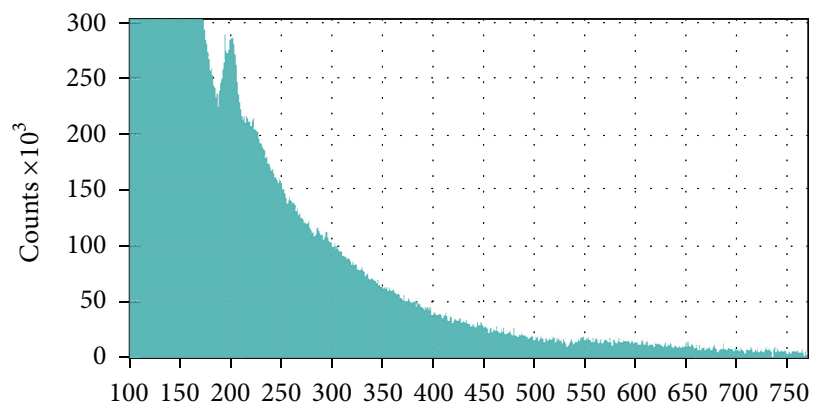

$(\mathrm{eV})$

(e)

FIGURE 6: (a) High magnification TEM image of boron nanotube. (b) Low magnification image of the same nanowire; note its growth is not constant. (c) Low magnification image of boron nanosheets and (d) high magnification image of the same material. The material appears to have folds and is amorphous in nature. (e) EELS spectrum for boron nanotubes and nanosheets, showing only boron is present. All materials in this image were grown with $\mathrm{MgB}_{2}$, no MCM-41, and an iron wire instead of nano-NiB.

could possess interesting properties. Future experiments could focus on methods to increase the yield of boron nanosheets and boron nanotubes. As of now, the effect of temperature, reaction time, catalyst, and so forth is not well understood. Another, even more exciting possibility beyond these homogeneous nanostructures would be the formation of boron-based nanoheterostructures, particularly with boron nitride and carbon. The boron nanosheets amorphous nature lends them to this type of application, as it eliminates issues with crystal mismatch. All of these experiments described in this paper were conducted in an inert atmosphere, but the addition of different reactant gases can result in various 


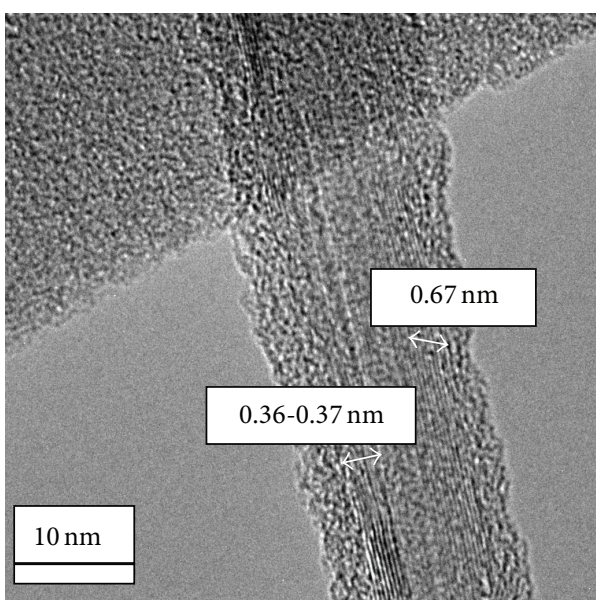

(a)

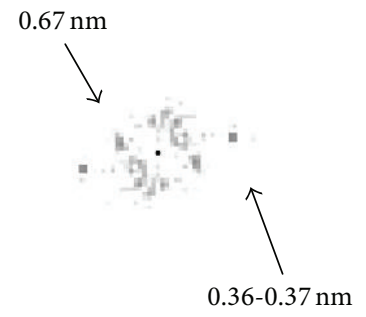

(b)

FIGURE 7: (a) High quality boron nanotube which was analyzed using fast Fourier transform (FFT) analysis. Note the structure is clearly hollow and the wall to wall spacing is $0.36-0.37 \mathrm{~nm}$. (b) FFT image used for analysis (image is $3 \mathrm{~nm} \times 3 \mathrm{~nm}$ ), confirming the interwall spacing is $0.36-0.37 \mathrm{~nm}$. All materials in this image were grown with $\mathrm{MgB}_{2}$, an iron wire instead of $\mathrm{NiB}$, and no MCM-41.

nanostructures [27]. The intermittent use of reactant gases could be an intriguing approach to create nanoheterostructures.

\section{Conflict of Interests}

The authors have no conflict of interests to disclose.

\section{References}

[1] S. Iijima, "Helical microtubules of graphitic carbon," Nature, vol. 354, no. 6348, pp. 56-58, 1991.

[2] M. S. Dresselhaus, G. Dresselhaus, J. C. Charlier, and E. Hernández, "Electronic, thermal and mechanical properties of carbon nanotubes," Philosophical Transactions of the Royal Society A: Mathematical, Physical and Engineering Sciences, vol. 362, no. 1823, pp. 2065-2098, 2004.

[3] M. Karwa, Z. Iqbal, and S. Mitra, "Scaled-up self-assembly of carbon nanotubes inside long stainless steel tubing," Carbon, vol. 44 , no. 7, pp. 1235-1242, 2006.

[4] R. B. Patel, J. Liu, J. V. Scicolone et al., "Formation of stainless steel-carbon nanotube composites using a scalable chemical vapor infiltration process," Journal of Materials Science, vol. 48, no. 3, pp. 1387-1395, 2013.

[5] A. K. Geim and K. S. Novoselov, "The rise of graphene," Nature Materials, vol. 6, no. 3, pp. 183-191, 2007.

[6] K. S. Novoselov, A. K. Geim, S. V. Morozov et al., "Electric field in atomically thin carbon films," Science, vol. 306, no. 5696, pp. 666-669, 2004.

[7] C. Mattevi, H. Kim, and M. Chhowalla, "A review of chemical vapour deposition of graphene on copper," Journal of Materials Chemistry, vol. 21, no. 10, pp. 3324-3334, 2011.

[8] R. Ma, Y. Bando, and T. Sato, "CVD synthesis of boron nitride nanotubes without metal catalysts," Chemical Physics Letters, vol. 337, no. 1-3, pp. 61-64, 2001.

[9] D. Golberg, Y. Bando, M. Mitome, K. Fushimi, and C. Tang, "Boron nitride nanotubes as nanocrucibles for morphology and phase transformations in encapsulated nanowires of the $\mathrm{Mg}-\mathrm{O}$ system," Acta Materialia, vol. 52, no. 11, pp. 3295-3303, 2004.

[10] R. B. Patel, J. Liu, J. Eng, and Z. Iqbal, “One-step CVD synthesis of a boron nitride nanotube-iron composite," Journal of Materials Research, vol. 26, no. 10, pp. 1332-1339, 2011.

[11] T. Kasuga, M. Hiramatsu, A. Hoson, T. Sekino, and K. Niihara, "Formation of titanium oxide nanotube," Langmuir, vol. 14, no. 12, pp. 3160-3163, 1998.

[12] Y. Feldman, E. Wasserman, D. J. Srolovitz, and R. Tenne, "Highrate, gas-phase growth of $\mathrm{MoS}_{2}$ nested inorganic fullerenes and nanotubes," Science, vol. 267, no. 5195, pp. 222-225, 1995.

[13] M. Nath, A. Govindaraj, and C. N. R. Rao, "Simple synthesis of $\mathrm{MoS}_{2}$ and $\mathrm{WS}_{2}$ nanotubes," Advanced Materials, vol. 13, no. 4, pp. 283-286, 2001.

[14] G. Radovsky, R. Popovitz-Biro, M. Staiger et al., "Synthesis of copious amounts of $\mathrm{SnS}_{2}$ and $\mathrm{SnS}_{2} / \mathrm{SnS}$ nanotubes with ordered superstructures," Angewandte Chemie, vol. 50, no. 51, pp. 1231612320, 2011.

[15] R. M. Wang, Y. J. Xing, J. Xu, and D. P. Yu, "Fabrication and microstructure analysis on zinc oxide nanotubes," New Journal of Physics, vol. 5, pp. 115.1-115.7, 2003.

[16] D. Ciuparu, R. F. Klie, Y. Zhu, and L. Pfefferle, "Synthesis of pure boron single-wall nanotubes," The Journal of Physical Chemistry B, vol. 108, no. 13, pp. 3967-3969, 2004.

[17] J. Kunstmann and A. Quandt, "Broad boron sheets and boron nanotubes: an ab initio study of structural, electronic, and mechanical properties," Physical Review B: Condensed Matter and Materials Physics, vol. 74, no. 3, Article ID 035413, 2006.

[18] J. Kunstmann and A. Quandt, "Constricted boron nanotubes," Chemical Physics Letters, vol. 402, no. 1-3, pp. 21-26, 2005.

[19] I. Cabria, M. J. López, and J. A. Alonso, "Density functional calculations of hydrogen adsorption on boron nanotubes and boron sheets," Nanotechnology, vol. 17, no. 3, pp. 778-785, 2006.

[20] X. Yang, Y. Ding, and J. Ni, "Ab initio prediction of stable boron sheets and boron nanotubes: structure, stability, and electronic properties," Physical Review B-Condensed Matter and Materials Physics, vol. 77, no. 4, Article ID 041402, 2008. 
[21] J. Liu and Z. Iqbal, "Facile synthesis of pure boron nanotubes and nanofibers," in MRS Proceedings, pp. 42-47, December 2010.

[22] J. J. Fu, Y. N. Lu, H. Xu et al., "The synthesis of boron nitride nanotubes by an extended vapour-liquid-solid method," Nanotechnology, vol. 15, no. 7, pp. 727-730, 2004.

[23] Z. Hu, Y. Fan, F. Chen, and Y. Chen, "Amorphous iron-boron powders prepared by chemical reduction of mixed-metal cation solutions: dependence of composition upon reaction temperature," Journal of the Chemical Society, Chemical Communications, no. 2, p. 247, 1995.

[24] L. M. Cao, Z. Zhang, L. L. Sun et al., "Well-aligned boron nanowire arrays," Advanced Materials, vol. 13, no. 22, pp. 17011704, 2001.

[25] F. Liu, J. Tian, L. Bao et al., "Fabrication of vertically aligned single-crystalline boron nanowire arrays and investigation of their field-emission behavior," Advanced Materials, vol. 20, no. 13, pp. 2609-2615, 2008.

[26] L. M. Cao, K. Hahn, C. Scheu et al., “Template-catalyst-free growth of highly ordered boron nanowire arrays," Applied Physics Letters, vol. 80, no. 22, pp. 4226-4228, 2002.

[27] R. B. Patel, Synthesis and characterization of novel boron-based nanostructures and composites [Dissertation], New Jersey Institute of Technlogy, New York, NY, USA, 2013. 

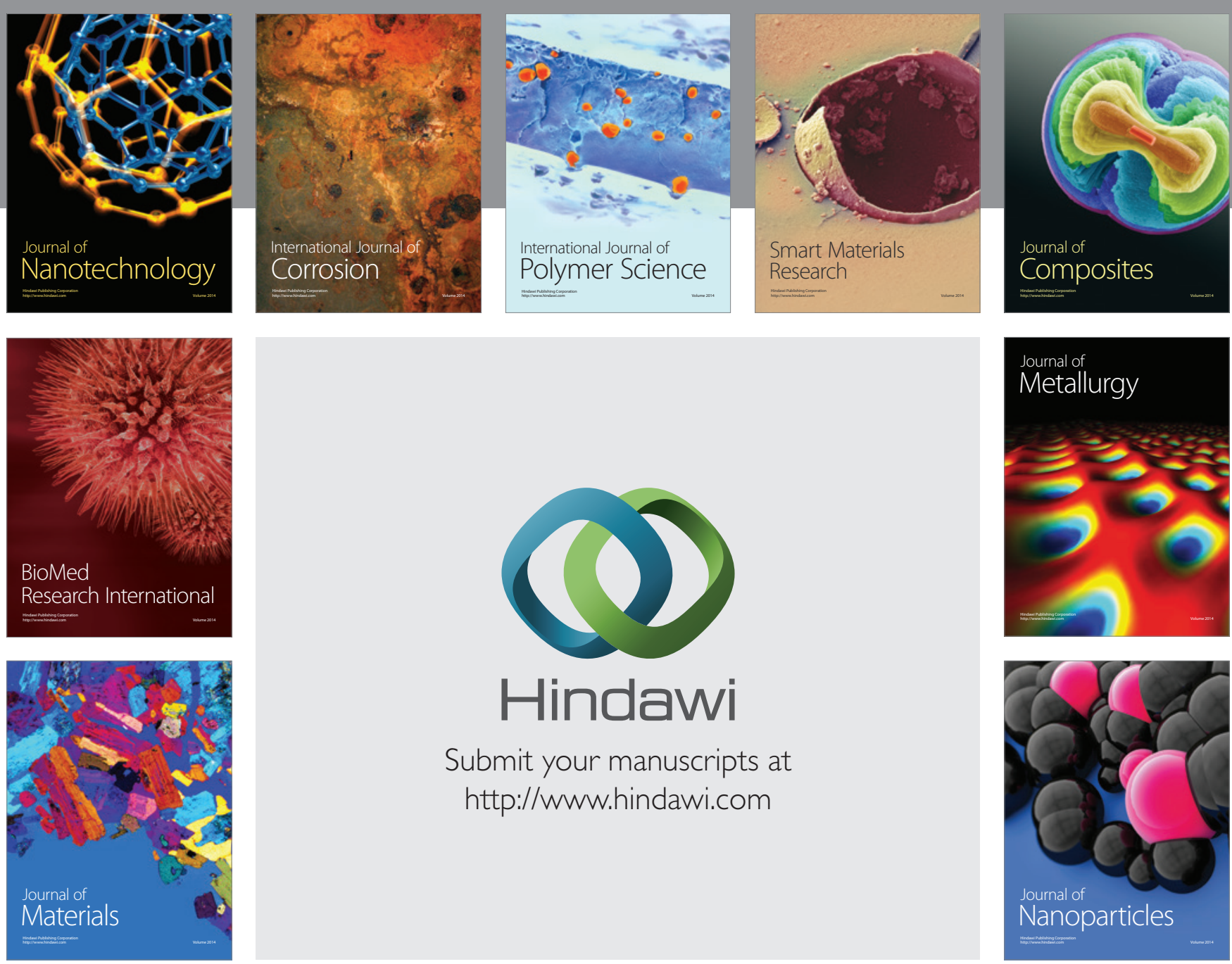

Submit your manuscripts at http://www.hindawi.com
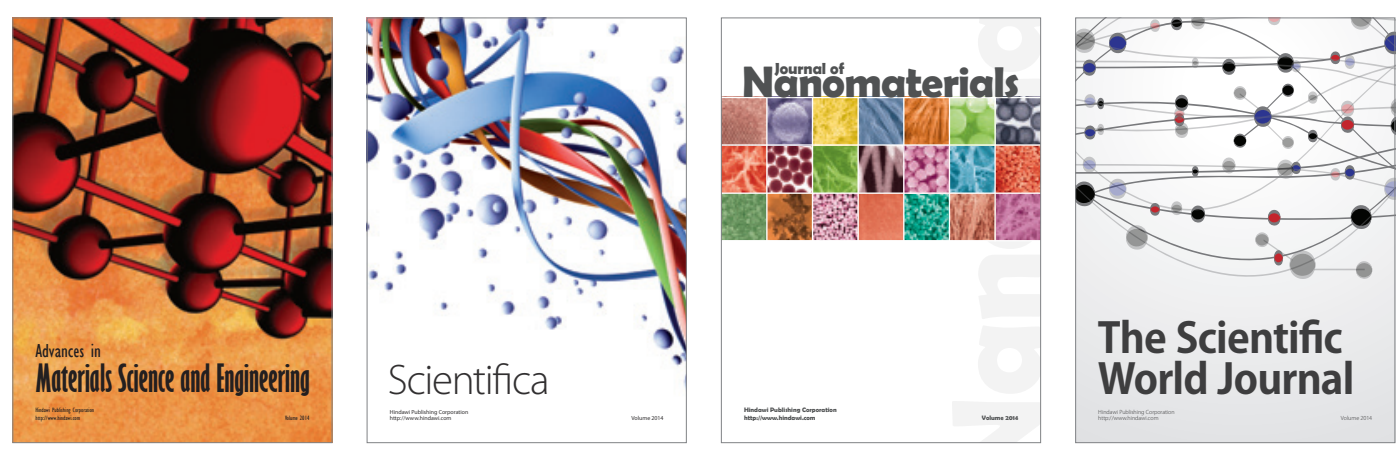

\section{The Scientific World Journal}
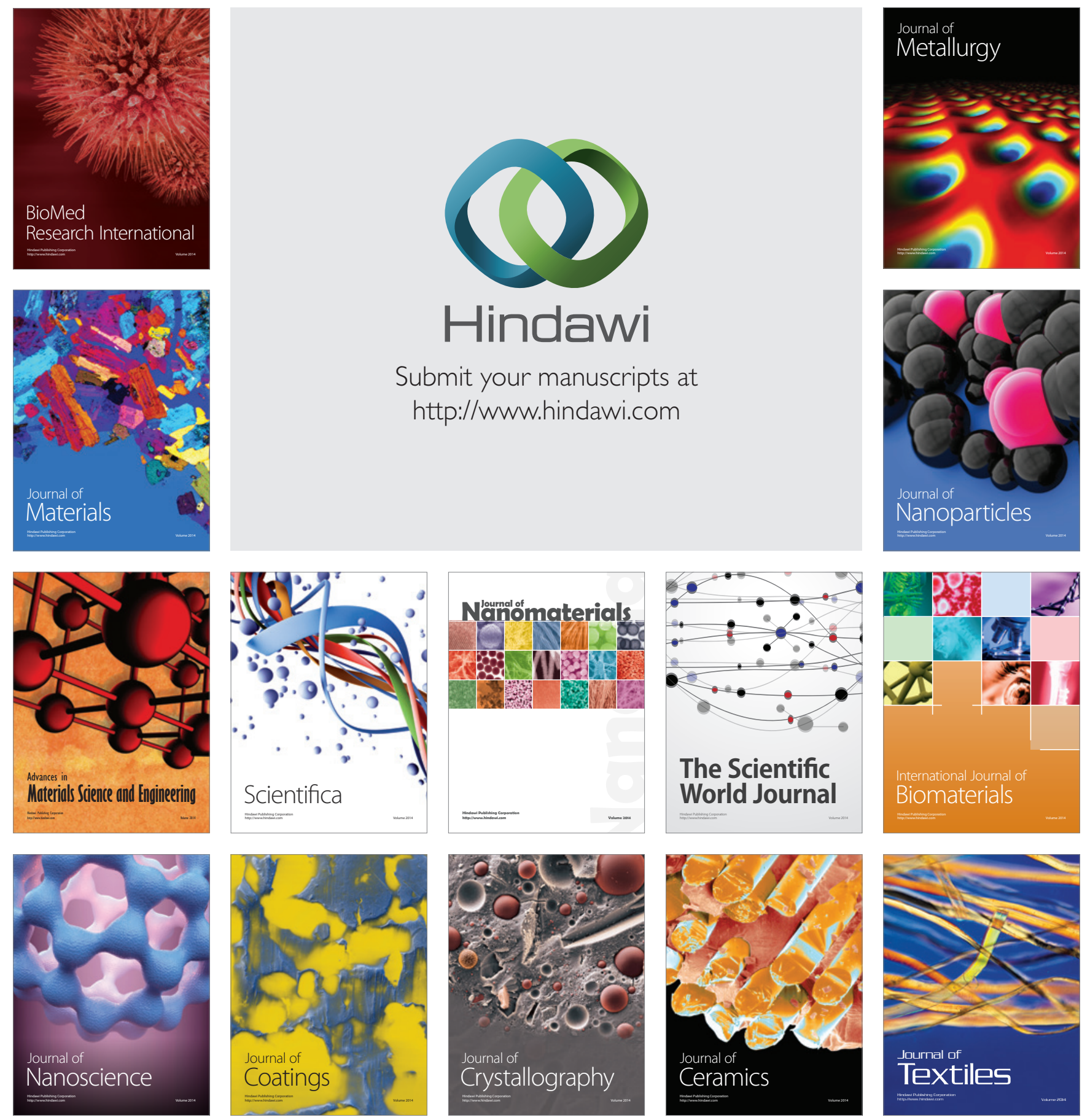